\title{
Case Report \\ Biopsy-Proven Acute Tubular Necrosis due to Vancomycin Toxicity
}

\author{
Farheen Shah-Khan, ${ }^{1}$ Marc H. Scheetz, ${ }^{2,3}$ and Cybele Ghossein ${ }^{1}$ \\ ${ }^{1}$ Division of Nephrology, Northwestern Memorial Hospital, Chicago, IL 60611, USA \\ ${ }^{2}$ Department of Pharmacy Practice, College of Pharmacy, Midwestern University Chicago, Downers Grove, IL 60515, USA \\ ${ }^{3}$ Department of Pharmacy, Northwestern Memorial Hospital, Chicago, IL 60611, USA
}

Correspondence should be addressed to Cybele Ghossein, c-ghossein@northwestern.edu

Received 28 February 2011; Accepted 26 April 2011

Academic Editor: Franca Anglani

Copyright ( $) 2011$ Farheen Shah-Khan et al. This is an open access article distributed under the Creative Commons Attribution License, which permits unrestricted use, distribution, and reproduction in any medium, provided the original work is properly cited.

Vancomycin (VAN) has been associated with acute kidney injury (AKI) since it has been put into clinical use in the 1950's. Early reports of AKI were likely linked to the impurities of the VAN preparation. With the advent of the more purified forms of VAN, the incidence of AKI related to VAN were limited to acute interstitial nephritis (AIN) or as a potentiating agent to other nephrotoxins such as Aminoglycosides. VAN as the sole etiologic factor for nephrotoxic acute tubular necrosis (ATN) has not been described. Here, we report a case of biopsy-proven ATN resulting from VAN.

\section{Case}

R. H. is a 23-year-old male with a history of childhood acute lymphocytic leukemia in remission, neuroectodermal tumor status postresection, and gamma knife therapy, who presented to our Emergency Department (ED) with complaints of fever and chills. He also noticed yellowish drainage around the site of a peripherally inserted central catheter (PICC). In the ED, patient was febrile to $101.7^{\circ}$ Fahrenheit with a blood pressure of $113 / 83 \mathrm{~mm} \mathrm{Hg}$ and pulse of $134 \mathrm{bpm}$. The PICC line was removed, and antibiotic therapy initiated with piperacillin-tazobactam and VAN. RH received $1 \mathrm{gm}$ IV of VAN in the ED, and upon admission to the floor, received another $2 \mathrm{gm}$ IV. Piperacillin-tazobactam was discontinued. Eight hours later, another 2 grams of VAN were given, with cumulative dose of $5 \mathrm{gm}$ in $24 \mathrm{hrs}$ (50 mg/kg in 24 hours). Patient's admission creatinine was $0.97 \mathrm{mg} / \mathrm{dL}$. The patient was $103 \mathrm{~kg}$ and $72^{\prime \prime}$ tall. Attempting to use a loading dose of $15 \mathrm{mg} / \mathrm{kg}$ based on total body weight [1], the patient was initiated on $2000 \mathrm{mg}$ of VAN per hospital protocol of using VAN doses in $1000 \mathrm{mg}$ aliquots. Standard pharmacokinetic equations with simplified onecompartment models employing log-linear drug removal were used for estimations [2]. With a volume of distribution estimated at approximately $0.6 \mathrm{~L} / \mathrm{kg}$ of actual body weight and a VAN clearance estimated to be at least $120 \mathrm{~mL} / \mathrm{min}$ [3], the patient was expected to achieve an initial VAN trough of approximately $8 \mathrm{mg} / \mathrm{L}$ with steady state troughs above $10 \mathrm{mg} / \mathrm{L}$ with a dosing scheme of $2000 \mathrm{mg}$ given every 12 hours. As pharmacokinetic estimates in patients above their average body weight exhibit substantial variability, the patient was initiated on the aforementioned dose with a measured VAN trough planned antecedent to the fourth dose (to capture steady-state concentrations). Goal troughs and monitoring schemes were performed according to national VAN guidelines [4].

The following day, the serum creatinine was $3.62 \mathrm{mg} / \mathrm{dL}$. He became oligo-anuric with a urine output of $50 \mathrm{cc}$ in 24 hours. Urinalysis revealed a bland urine sediment and a urine sodium of $75 \mathrm{meq} / \mathrm{L}$ and a fractional excretion of sodium of $2.77 \%$. He had not been exposed to IV contrast, received an aminoglycoside or exposed to any other potential nephrotoxins.

Blood cultures were negative, and the PICC line tip cultures were positive for Serratia marcescens. As the patient experienced acute nephrotoxicity, VAN was ceased after the third dose and ciprofloxacin initiated. The creatinine continued to rise (Table 1). A renal ultrasound ruled out 


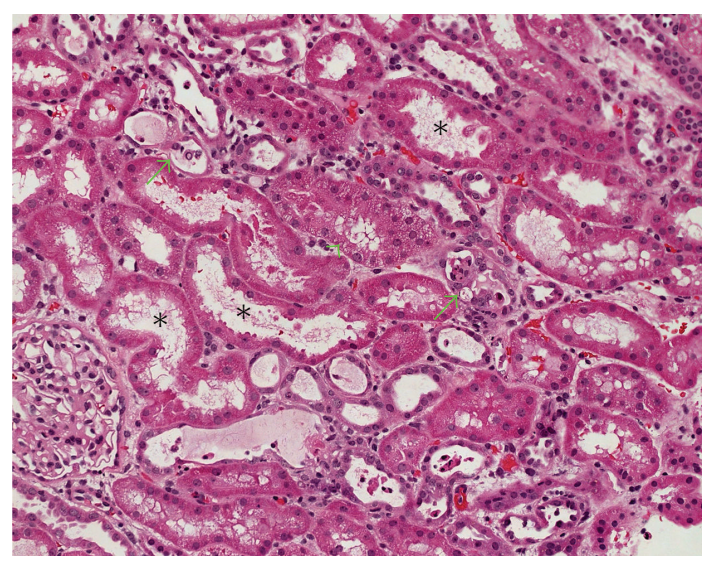

(a)

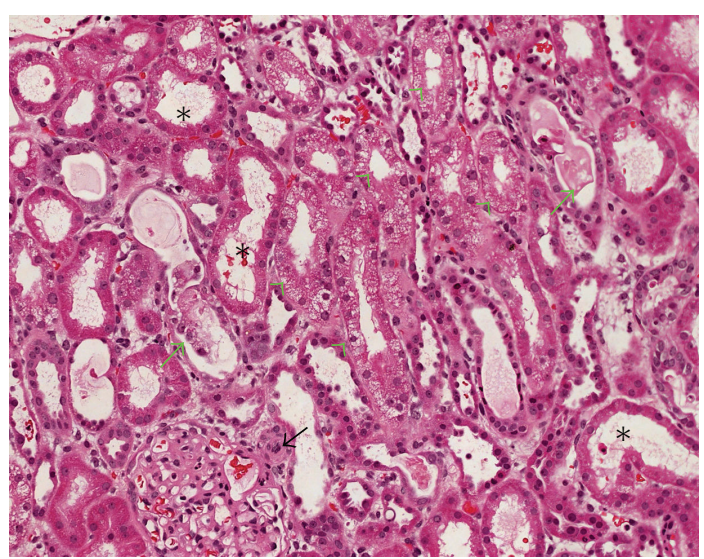

(b)

FiguRE 1: Representative photographs of the renal biopsy showing tubular damage secondary to drug toxicity. In both panels (a) and (b) a number of tubules show moderate degree of acute tubular necrosis (asterisks). Some of tubules contain hyaline or epithelial casts in their lumina (green arrows), while several tubules show vacuolization of their cytoplasm (green arrowheads). One of the glomerular afferent arteriole shows swollen endothelia and occlusive change (black arrow).

TABLE 1: Laboratory and clinical parameters.

\begin{tabular}{lccccc}
\hline Day & Scr mg/dl & UO $24 \mathrm{hrs}$ & VAN dose & VAN S. Conc. & HD \\
\hline 0 & .97 & NR & $5 \mathrm{gm}$ & ND & No \\
1 & $3.62-4.26$ & $50 \mathrm{cc}$ & & ND & No \\
2 & 6.25 & $<50 \mathrm{cc}$ & & ND & No \\
3 & 8.41 & $<50 \mathrm{cc}$ & & ND & No \\
4 & 9.96 & $<50 \mathrm{cc}$ & 64.7 & Yes \\
5 & 9.36 & $1000 \mathrm{cc}$ & & Yes \\
9 & 9.21 & $2000 \mathrm{cc}$ & $\mathrm{ND}$ & Yes \\
10 & 5.88 & $2500 \mathrm{cc}$ & & ND & No \\
30 & 1.24 & $\mathrm{NR}$ & & ND & No \\
\hline
\end{tabular}

Scr—serum creatinine; UO-urine output; VAN—Vancomycin; S. ConcSerum concentration; HD—hemodialysis; ND—not done; NR—not recorded.

obstruction. On day 3, a renal biopsy was performed. The main finding was ATN (Figure 1). Many tubules showed moderate degree of vacuolization of their cytoplasm (green arrows). Some of tubules contained hyaline or epithelial casts in their lumina (black arrows). The VAN serum concentration done on day 4 was $64.7 \mathrm{mg} / \mathrm{L}$, and hemodialysis was initiated for volume overload. By day 5, his urine output increased to $1 \mathrm{~L} /$ day. On day 10 , he was taken off hemodialysis. His creatinine improved to $1.2 \mathrm{mg} / \mathrm{dL}$ over the next few weeks.

\section{Discussion}

VAN, a parenteral glycopeptide antibiotic, has been used clinically since 1956 . The original preparation contained a number of impurities and was brown in color, hence, the nickname "Mississippi Mud". It is these impurities that were thought to be responsible for certain toxicities such as anaphylaxis, toxic epidermal necrolysis, erythema multiforme, ototoxicity, and nephrotoxicity [5, 6]. Between 1956 and 1986, 57 cases of VAN-associated nephrotoxicity were described. More than $50 \%$ of the cases were identified within the first six years of VAN use when the product was relatively impure [7]. As manufacturing processes improved and VAN was purified, nephrotoxicity became less common [8], and contemporary preparations seemed to nearly eliminate the original adverse events.

In 1983, Farber andMoellering reported the incidence of nephrotoxicity with VAN to be 5\% [5]. This, however, was a retrospective study, and it failed to evaluate whether the AKI was due to AIN or ATN. VAN in association with an aminoglycoside is known to be associated with an increased incidence of nephrotoxicity presumed to be ATN $[9,10]$.

Other risk factors associated with nephrotoxicity include prolonged therapy for $>21$ days, [9] higher APACHE scores, [11] loop diuretics, and steady-state VAN concentration > or $=28 \mathrm{mg} / \mathrm{L}$ [12]. Recent studies have shown that doses greater than or equal to 4 grams per day [13] and trough concentrations greater than $15 \mathrm{mg} / \mathrm{L}$ are associated with nephrotoxicity $[11,14]$. More specifically, when trough concentrations are analyzed as a linear variable with classification and regression tree modeling, a threshold of $9.9 \mathrm{mg} / \mathrm{L}$ has been identified [15]. These studies, while instructive for the association of VAN and toxicity, are limited by a failure to control for weight-adjusted dose, and the retrospective nature of the studies do not determine causality $[11,13-$ 15]. Instead, elevated VAN concentrations could be merely an intermediate variable in the pathway to the ultimate causal event of renal failure by other mechanisms.

As VAN safety profile improved and with the emerging prevalence of methicillin-resistant Staphylococcus aureus (MRSA), VAN became one of the most frequently prescribed intravenous anti-infective for treatment of these infections [16]. With increasing the minimum inhibitory concentrations for VAN and proper testing methodologies for completing minimum inhibitory concentrations under continual debate [17-20], the dire outcomes associated with 
MRSA infections have lead many to seek novel treatment strategies including increasing VAN doses and exposure. Specifically, various national guidelines for serious infections have recommended that VAN troughs be maintained between $15-20 \mathrm{mg} / \mathrm{L}$ to ensure an AUC/MIC of $\sim 400$ [2123]. Such a position has been substantiated on the basis of appropriate pharmacodynamic targets, limited clinical data, and the supposition that such a strategy is safe [21, 22, 24]. However, no study to date has prospectively evaluated the impact of VAN exposure on renal endpoints and that elevated doses (such as a 5 gram dose given in 24 hours in our patient) could predispose patients to kidney injury.

The potential mechanism of action of VAN-associated nephropathy has been studied in both humans and animals. The energy-dependent transport mechanisms found in the proximal tubular epithelium render the kidneys highly susceptible to toxicant-induced renal injury. VAN exposure in renal proximal tubule epithelial cells results in increased cell proliferation as evidenced by increased number of cells, total protein, and DNA synthesis. VAN enhances cellular ATP concentration and stimulates oxygen consumption, supporting its role as a stimulant of oxidative phosphorylation [25]. The beneficial effect of some antioxidants like DL- $\alpha$ lipoic acid, Melatonin, Ginkgo biloba and milrinone have been shown to reduce the renal damage, suggesting the involvement of free radicals in renal damage [26].

While it is unclear if VAN-associated acute tubular necrosis is preventable, these data argue for prompt serum VAN monitoring given the rapid functional decline of patient nephrologic function even before the patient was scheduled to receive the 4th dose of therapeutically dosed VAN. Additionally, these data may support previously published literature suggesting that VAN doses $>4 \mathrm{~g}$ per day, and patient body weights in excess of $101.4 \mathrm{~kg}$ may predispose to nephrotoxicity, [27] in this case, acute tubular necrosis.

\section{Conclusion}

The most common VAN-associated nephrotoxicity is AIN [28-30]. Although an association with ATN has been described, to our knowledge, VAN as the sole etiologic cause of ATN has never been reported in adult patients [31, 32]. Our findings support these retrospective associations and provide evidence for contemporary VAN-associated ATN. Further studies are needed to determine the safety profile of targeting higher VAN trough levels for serious infectious in light of the potential for AKI.

\section{References}

[1] K. Vance-Bryan, D. R. P. Guay, S. S. Gilliland, K. A. Rodvold, and J. C. Rotschafer, "Effect of obesity on vancomycin pharmacokinetic parameters as determined by using a Bayesian forecasting technique," Antimicrobial Agents and Chemotherapy, vol. 37, no. 3, pp. 436-440, 1993.

[2] M. E. Winter, Basic Clinical Pharmacokinetics, Lippincott Williams \& Wilkins, Philadelphia, Pa, USA, 4th edition, 2003.
[3] D. T. Bearden and K. A. Rodvold, "Dosage adjustments for antibacterials in obese patients: applying clinical pharmacokinetics," Clinical Pharmacokinetics, vol. 38, no. 5, pp. 415-426, 2000.

[4] M. Rybak, B. Lomaestro, J. C. Rotschafer et al., "Therapeutic monitoring of vancomycin in adult patients: a consensus review of the American Society of Health-System Pharmacists, the Infectious Diseases Society of America, and the Society of Infectious Diseases Pharmacists," American Journal of HealthSystem Pharmacy, vol. 66, no. 1, pp. 82-98, 2009.

[5] B. F. Farber and R. C. Moellering Jr., "Retrospective study of the toxicity of preparations of vancomycin from 1974 to 1981," Antimicrobial Agents and Chemotherapy, vol. 23, no. 1, pp. 138-141, 1983.

[6] D. W. Woodley and W. H. Hall, "The treatment of severe staphylococcal infections with vancomycin," Annals of Internal Medicine, vol. 55, pp. 235-249, 1961.

[7] G. R. Bailie and D. Neal, "Vancomycin ototoxicity and nephrotoxicity. A review," Medical Toxicology and Adverse Drug Experience, vol. 3, no. 5, pp. 376-386, 1988.

[8] D. P. Levine, "Vancomycin: a history," Clinical Infectious Diseases, vol. 42, supplement 1, pp. S5-S12, 2006.

[9] M. B. Goetz and J. Sayers, "Nephrotoxicity of vancomycin and aminoglycoside therapy separately and in combination," Journal of Antimicrobial Chemotherapy, vol. 32, no. 2, pp. 325334, 1993.

[10] M. J. Rybak, B. J. Abate, S. L. Kang, M. J. Ruffing, S. A. Lerner, and G. L. Drusano, "Prospective evaluation of the effect of an aminoglycoside dosing regimen on rates of observed nephrotoxicity and ototoxicity," Antimicrobial Agents and Chemotherapy, vol. 43, no. 7, pp. 1549-1555, 1999.

[11] M. N. Jeffres, W. Isakow, J. A. Doherty, S. T. Micek, and M. H. Kollef, "A retrospective analysis of possible renal toxicity associated with vancomycin in patients with health care-associated methicillin-resistant Staphylococcus aureus pneumonia," Clinical Therapeutics, vol. 29, no. 6, pp. 11071115, 2007.

[12] L. S. Elting, E. B. Rubenstein, D. Kurtin et al., "Mississippi mud in the 1990s: risks and outcomes of vancomycin- associated toxicity in general oncology practice," Cancer, vol. 83, no. 12, pp. 2597-2607, 1998.

[13] T. P. Lodise, B. Lomaestro, J. Graves, and G. L. Drusano, "Larger vancomycin doses (at least four grams per day) are associated with an increased incidence of nephrotoxicity," Antimicrobial Agents and Chemotherapy, vol. 52, no. 4, pp. 1330-1336, 2008.

[14] L. K. Hidayat, D. I. Hsu, R. Quist, K. A. Shriner, and A. WongBeringer, "High-dose vancomycin therapy for methicillinresistant Staphylococcus aureus infections: efficacy and toxicity," Archives of Internal Medicine, vol. 166, no. 19, pp. 21382144, 2006.

[15] T. P. Lodise, N. Patel, B. M. Lomaestro, K. A. Rodvold, and G. L. Drusano, "Relationship between initial vancomycin concentration-time profile and nephrotoxicity among hospitalized patients," Clinical Infectious Diseases, vol. 49, no. 4, pp. 507-514, 2009.

[16] H. A. Kirst, D. G. Thompson, and T. I. Nicas, "Historical yearly usage of vancomycin," Antimicrobial Agents and Chemotherapy, vol. 42, no. 5, pp. 1303-1304, 1998.

[17] E. O. Mason, L. B. Lamberth, W. A. Hammerman, K. G. Hulten, J. Versalovic, and S. L. Kaplan, "Vancomycin MICs for Staphylococcus aureus vary by detection method and have 
subtly increased in a pediatric population since 2005," Journal of Clinical Microbiology, vol. 47, no. 6, pp. 1628-1630, 2009.

[18] H. S. Sader, P. R. Rhomberg, and R. N. Jones, "Nine-hospital study comparing broth microdilution and Etest method results for vancomycin and daptomycin against methicillinresistant Staphylococcus aureus," Antimicrobial Agents and Chemotherapy, vol. 53, no. 7, pp. 3162-3165, 2009.

[19] J. M. Swenson, K. F. Anderson, D. R. Lonsway et al., "Accuracy of commercial and reference susceptibility testing methods for detecting vancomycin-intermediate Staphylococcus aureus," Journal of Clinical Microbiology, vol. 47, no. 7, pp. 2013-2017, 2009.

[20] H. S. Sader, P. D. Fey, D. N. Fish et al., "Evaluation of vancomycin and daptomycin potency trends (MIC creep) against methicillin-resistant Staphylococcus aureus isolates collected in nine U.S. Medical Centers from 2002 to 2006," Antimicrobial Agents and Chemotherapy, vol. 53, no. 10, pp. 4127-4132, 2009.

[21] M. J. Rybak, B. M. Lomaestro, J. C. Rotscahfer et al., "Vancomycin therapeutic guidelines: a summary of consensus recommendations from the infectious diseases Society of America, the American Society of Health-System Pharmacists, and the Society of Infectious Diseases Pharmacists," Clinical Infectious Diseases, vol. 49, pp. 325-327, 2009.

[22] L. A. Mandell, R. G. Wunderink, A. Anzueto et al., "Infectious Diseases Society of America/American Thoracic Society Consensus Guidelines on the management of communityacquired pneumonia in adults," Clinical Infectious Diseases, vol. 44, supplement 2, pp. S27-S72, 2007.

[23] A. R. Tunkel, B. J. Hartman, S. L. Kaplan et al., "Practice guidelines for the management of bacterial meningitis," Clinical Infectious Diseases, vol. 39, no. 9, pp. 1267-1284, 2004.

[24] M. H. Scheetz, R. G. Wunderink, M. J. Postelnick, and G. A. Noskin, "Potential impact of vancomycin pulmonary distribution on treatment outcomes in patients with methicillinresistant Staphylococcus aureus pneumonia," Pharmacotherapy, vol. 26, no. 4, pp. 539-550, 2006.

[25] D. W. King and M. A. Smith, "Proliferative responses observed following vancomycin treatment in renal proximal tubule epithelial cells," Toxicology in Vitro, vol. 18, no. 6, pp. 797-803, 2004.

[26] I. Celik, M. Cihangiroglu, N. Ilhan, N. Akpolat, and H. H. Akbulut, "Protective effects of different antioxidants and amrinone on vancomycin-induced nephrotoxicity," Basic and Clinical Pharmacology and Toxicology, vol. 97, no. 5, pp. 325332, 2005.

[27] T. P. Lodise, B. Lomaestro, J. Graves, and G. L. Drusano, "Larger vancomycin doses (at least four grams per day) are associated with an increased incidence of nephrotoxicity," Antimicrobial Agents and Chemotherapy, vol. 52, no. 4, pp. 1330-1336, 2008.

[28] M. M. Bergman, R. H. Glew, and T. H. Ebert, "Acute interstitial nephritis associated with vancomycin therapy," Archives of Internal Medicine, vol. 148, no. 10, pp. 2139-2140, 1988.

[29] C. E. Codding, L. Ramseyer, M. Allon, J. Pitha, and M. Rodriguez, "Tubulointerstitial nephritis due to vancomycin," American Journal of Kidney Diseases, vol. 14, no. 6, pp. 512$515,1989$.

[30] A. O. Wai, A. M. S. Lo, A. Abdo, and F. Marra, "Vancomycininduced acute interstitial nephritis," Annals of Pharmacotherapy, vol. 32, no. 11, pp. 1160-1164, 1998.
[31] E. Fiaccadori, U. Maggiore, A. Arisi et al., "Outbreak of acute renal failure due to cefodizime-vancomycin association in a heart surgery unit," Intensive Care Medicine, vol. 27, no. 11, pp. 1819-1822, 2001.

[32] H. Sokol, C. Vigneau, E. Maury, B. Guidet, and G. Offenstadt, "Biopsy-proven anuric acute tubular necrosis associated with vancomycin and one dose of aminoside," Nephrology Dialysis Transplantation, vol. 19, no. 7, pp. 1921-1922, 2004. 


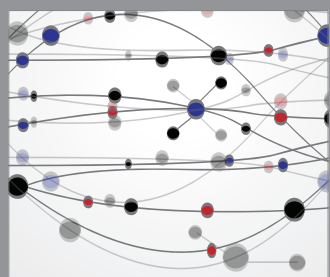

The Scientific World Journal
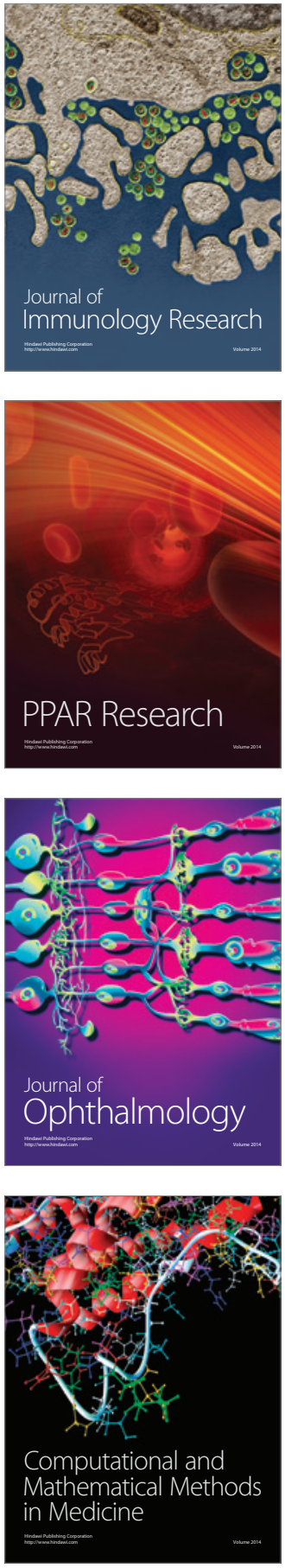

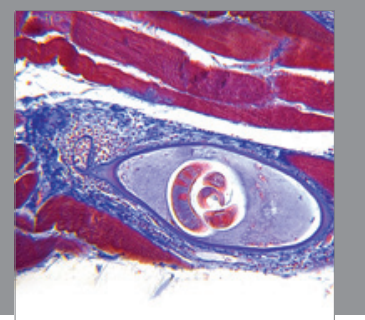

Gastroenterology

Research and Practice
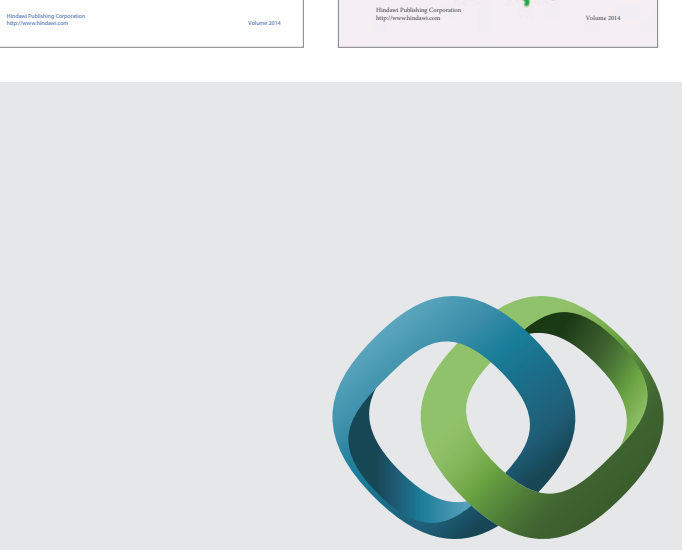

\section{Hindawi}

Submit your manuscripts at

http://www.hindawi.com
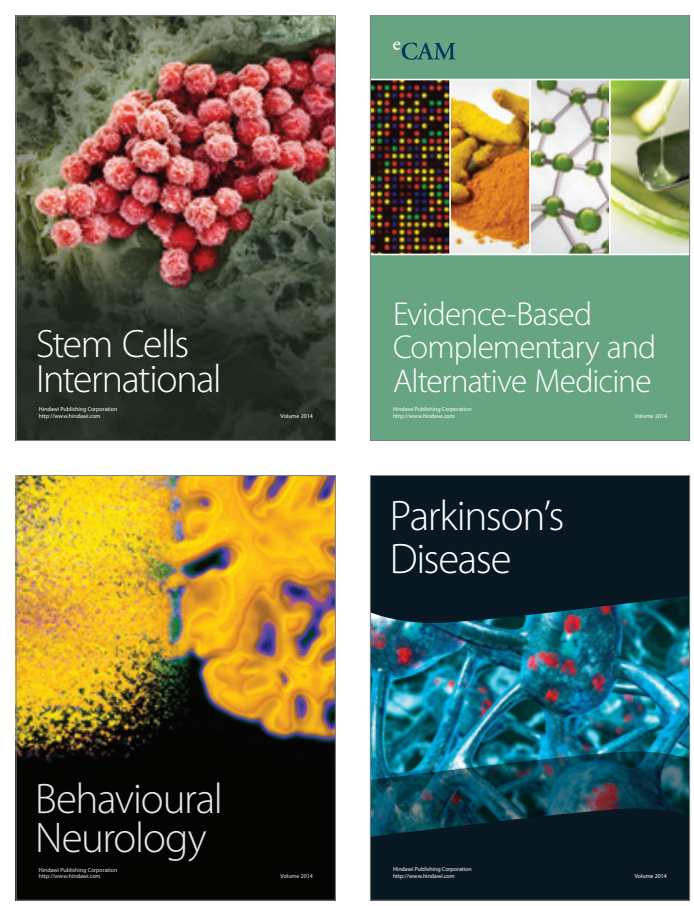

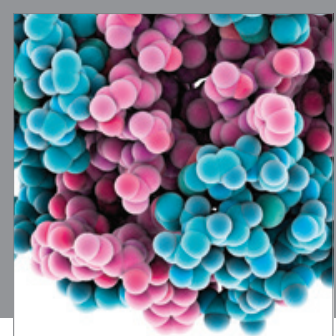

Journal of
Diabetes Research

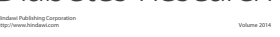

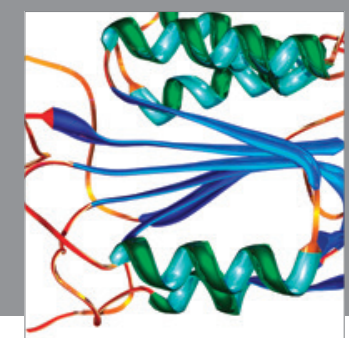

Disease Markers
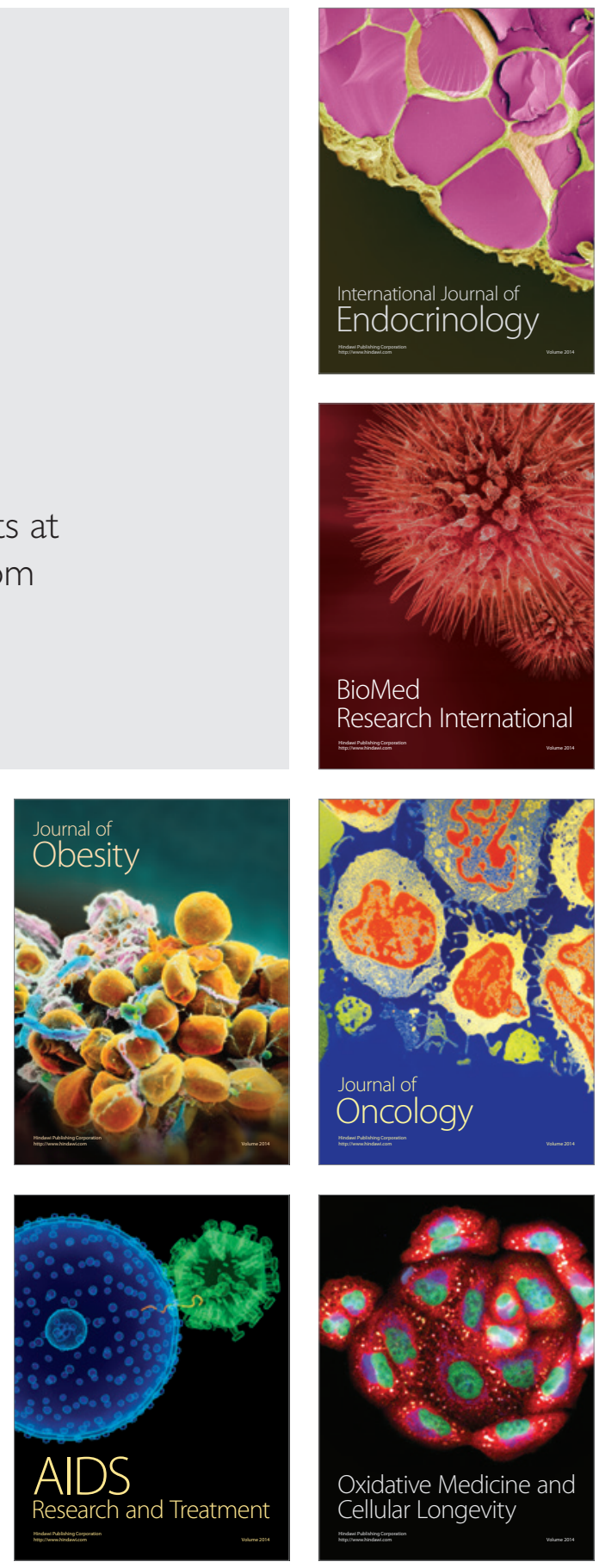